\title{
Tumor Identification in CT Medical Images using Semi Automatic Active Contour Models
}

\author{
${ }^{1}$ P.V.V.Kishore, ${ }^{2}$ Sampath Kumar, ${ }^{3}$ Y.Sahithi Reddy, ${ }^{4}$ J.K.Chaithanya, ${ }^{5}$ M.V.D.Prasad \\ 1, 2, 3, ${ }^{\mathrm{K}}$.L. University, Dept of E.C.E, KL University, Vaddeswaram, Green Fields, GUNTUR, AP, INDIA
}

\begin{abstract}
Damage of nerves occurs when an external force traumatically injures the nerves which cause a host of physical, cognitive and behavioral effects can range from complete recovery or permanent disability or death. The delineation of the trauma in brain, abdomen and lungs is a challenging task due to variations in shapes and image quality. In this method we present a semi automatic method for extracting the damage of nerves in brain, abdomen and lung from cardiac CT images, in which all the nerves are located sequentially. Each nerve is detected by first identifying the injuries and then segmenting the damaged part. To this end, the damaged nerve is localized by utilizing its geometric features obtained on-line from a CT image. After that, a variational region-growing model is employed to extract the damages. In particular, the location of the nerves damage is determined using via active contour model on the surface of the nerves. This extracts the damage of the nervous system and determines the exact accuracy of the damage is demonstrated by experimental results.
\end{abstract}

\section{Keywords}

Medical Image Diagnostics, Computer Tomography, Active Contours, Level sets, Tumor Detection.

\section{INTRODUCTION}

Medical imaging has played a vital role in diagnostics [1]-[4] by assisting doctors in disease identification. In this paper we want to introduce a new technology 'Active Contours (snakes)' method for Medical Applications. Generally injury occurs when an outside force traumatically injures the brain which cause a host of physical, cognitive and behavioral effects can range from complete mending or everlasting disability or death. The delineation of the trauma in brain, abdomen and lungs is a challenging task due to variations in shapes and image quality. In this method we propose an automatic method for extracting the damage in brain, abdomen and lung from cardiac CT Images. Each nerve is detected by first identifying the injuries and then segmenting the damaged part.

To this end, the damaged nerve is confined to a small area by employing its geometric features acquired on-line from a CT image. After that, a variational region-growing model is engaged to extract the damages. In particular, the location of the nerves damage is determined using via active Contour model on the surface of the nerves. This extracts the damage of the nervous system and determines the exact accuracy of the damage is demonstrated by experimental results. Generally we use CT images, MRI scans in order to find the TUMOR or to find out the damages occurs in the nerves. Whereas, to increase the quality and accuracy in finding the TUMOR's and Damages we are looking forward to suppose this Idea (method).This method includes the techniques like "ACTIVE CONTOUR" via shape segmentation[5]-[8].

Generally we use CT images, MRI scans in order to find the TUMOR or to find out the damages occurs in the nerves.
Whereas, to increase the quality and accuracy in finding the TUMOR's and damages we are looking forward to suppose this Idea(method).This method includes the techniques like "ACTIVE CONTOUR" and "SHAPE SEGMENTATION" where these techniques are helpful to extract the shape and quality of the image as CT image is applying as an input for this method. Therefore the extracted image quality and required area of identification for finding the TUMOR has been detected by using Geometrical comparison that which depends on the image whether it is brain or abdomen[9].

A CT scan [10] uses a computer that takes data from x-ray images of structures inside a human body and converts them into pictures on the monitor. Tomography is the process of generating a $2 \mathrm{D}$ image of a slice or section through 3D image. A CT scanner uses digital geometry processing to generate a 3D image inside the object. The 3D image is made after many $2 \mathrm{D} x$-ray images are taken around a single axis of rotation. Although CT is a useful tool for assisting diagnosis in medicine, it is a source of ionizing radiation and can cause cancer. The accuracy and speed of CT scanner may be improved by the application of spiral CT For our research we have obtained CT images from radiology lab at NRI Hospital, Guntur DT, Andhra Pradesh. Two of the abdomen images as shown in figure 1 .

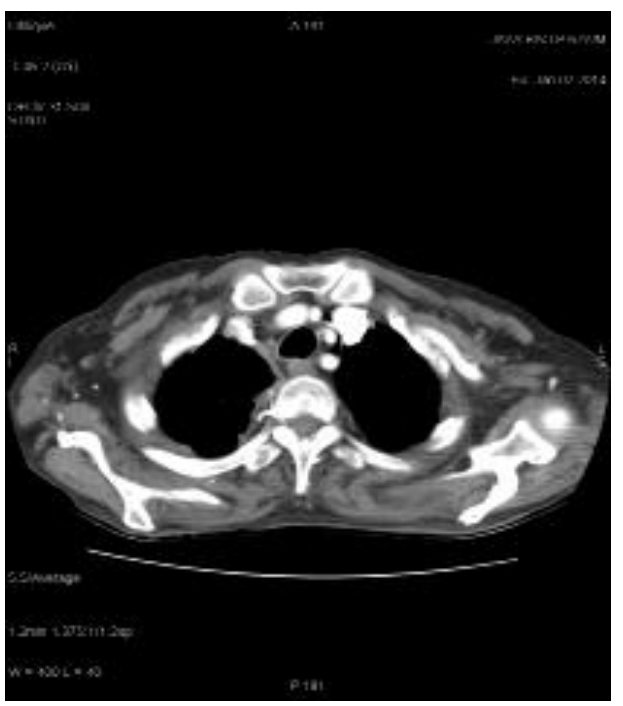

Figure 1: CT Abdomen Image

This problem is attacked by many researchers from around the globe by various algorithms such as background subtraction [11], texture color based segmentation [12] and deformable shape based segmentation [13]. In background subtraction [11] various algorithms have been proposed such as frame differencing, adaptive mean filtering, adaptive median filtering and Gaussian Mixture Models (GMM). Of all the proposed algorithms GMM gives good segmentation levels for all kinds of complex video backgrounds. But the main factor in GMM is the speed of operation which is very less compared to current standards. 
The algorithms discussed are not robust when applied to CT medical image tumor segmentation. To effectively segment CT medical image tumor and extract CT tumors we propose semi automatic active contours based CT segmentation and Tumor identification[15]-[17]. The rest of the paper is organized as follows. Section 2 deals with theory on active contour modes. Level sets a new approach to active contours[18]-[19] is proposed in section 3. Application of active contour models to CT image segmentation and tumor identification is presented in section 4. Section 5 presents results and discussion. Section 6 concludes the research results obtained in this work.

\section{THEORETICAL BACKGROUND- SNAKES THE FIRST MODELS}

The active contours are elastic models of continuous, flexible curve that is imposed upon and matched to the image by varying the elastic parameters. The fundamental idea is to make the curve or snake to fit tightly to the boundary of a particular object in the image. The design of evolution equation is such that the snake can easily

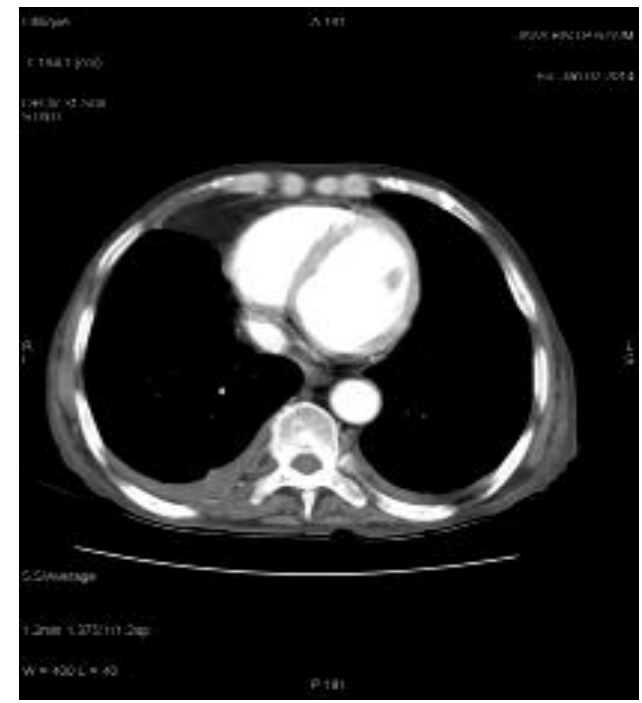

Figure 2: CT Abdomen Image at a Different Time Instance

embrace the object of importance, to be able to develop a similarity. The first snake model was proposed by kass [14]. The minimization energy function in order to achieve equilibrium is

$$
E^{\text {Snake }}=\int_{0}^{1}\left\{E^{\text {in }}(v(s))+E^{\text {image }}(v(s))\right\} d s
$$

represented parametrically by a planer curve

$$
v(s)=(x(s), y(s))
$$

and $E^{\text {in }}$ represents the internal energy of the curve due to bending and $E^{\text {image }}$ represents the image forces that push the snake towards the desired object.

The internal energy model was defined as

$$
E^{\text {in }}=\frac{\left(\alpha(s)\left|v^{\prime}(s)\right|^{2}+\beta(s)\left|v^{\prime \prime}(s)\right|^{2}\right)}{2}, \quad s \in[0,1]
$$

Where $v^{\prime}(s)$ First derivative of $v(s)$ and $v^{\prime \prime}(s)$ is Second order derivative of $v(s)$ with respect to $s$. The model of image energy is defined as

$$
E^{\text {image }}=-|\nabla I(x, y)|^{2}
$$

The first derivative of $v(s)$ with respect to ${ }^{\prime} s$, gives us rate of change of length of the curve. The coefficient $\alpha(s)$ allows the curve to have smaller or larger degree of contraction of the curve and therefore makes the snake act like an elastic string. The second derivative of $v(s)$ with respect to $s$, gives us rate of change of curvature. The coefficient $\beta(s)$ regulates the rate of the change of the curve in the direction normal to the boundary, preserving the smoothness of the curve. By adjusting these two coefficients, the curve gets an appropriate flexibility and is able to embrace the object of interest.

\section{THE LEVEL SET MODEL}

James A. Sethian and Stanley Osher technique as in [8] represented boundaries of $\Gamma(x)$ implicitly and model their propagation using appropriate partial differential equations. The boundary is given by level sets of a function $\phi(x)$. In level sets method, the interface boundary is characterize by a zero level set function $\phi(x)=0$, where $\phi: \mathfrak{R}^{2} \rightarrow \mathfrak{R} \quad \Gamma \quad$ is defined for al values of $x$

$$
\Gamma=\left\{\phi(x)=0, x \in \mathfrak{R}^{2}\right\}
$$

The sign of $\phi(x)$ defines whether $x$ is inside the contour $\Gamma$ or external to it. The sets defined as $\Gamma^{\text {int }}=\{x, \phi(x) \leq 0\}$ and $\Gamma^{\mathrm{ext}}=\{x, \phi(x) \geq 0\}$. The level set evolves based on the curvature of the image objects and assuming the curve moves towards the outward normal $\bar{n}$ defined in terms of parameter $\phi$ as

$$
\kappa=\nabla \cdot\left[\frac{\nabla \phi}{|\nabla \phi|}\right] \text { and } \bar{n}=\frac{\nabla \phi}{|\nabla \phi|}
$$

Usually the curve $\Gamma$ evolution is a time dependent process and the time dependent level set function $\phi: \mathfrak{R}^{2} \times \mathfrak{R} \rightarrow \mathfrak{R}$ as $\Gamma(t)=\left(\phi(x, t)=0, x \in \mathfrak{R}^{2}\right)$. One way to solve is to approximate spatial derivatives of motion and update the position of the curve over time. This method of solving the level sets is prone to unsteadiness due to erroneousness detection of position of the curve.

A different approach was proposed from the theory of level sets in [8]. Start with a zero level set $\phi(x)=0$ of higher dimension function and entrench the object curvature. Initializing the level set function $\phi_{\text {at }} t=0$, we have

$$
\phi(x, t=0)= \pm d
$$

Where ' $d$ ' is signed distance function (sdf) from $x$ to the curvature of the image object. If $d$ is a positive value $x$ is 
outside the object boundary and if $d$ is a negative value $x$ is inside the object boundary. The goal is to construct an equation for evolution of $\phi(x, t)$ to embrace the object boundaries from zero level set $\phi(x)=0$

We can propagate the zero level set $\phi(x)=0$, by solving a convection equation containing the velocity field ${ }^{v}$, which propagates all the level sets as

The $\phi_{t}+v \cdot \nabla \phi=0 \begin{gathered}\text { (8) } \\ \text { motion is normal velocity of the }\end{gathered}$
\[ v=F \bar{n}=F \frac{\nabla \phi}{|\nabla \phi|} \]
curve which is given by eq.6 as eq. 8 we have a level set equation of the form

$$
\phi_{t}+F|\nabla \phi|=0
$$

Eq.9 is a type of Hamilton-Jacobi

equation. The speed term $F$ is $\kappa=\nabla \cdot\left[\frac{\nabla \phi}{|\nabla \phi|}\right]$

dependent of object curvature, which can be formulated as

$$
F(\kappa)=F_{0}+F_{1}(F)
$$

Eq.10 drives the contour to level out with the high curvature regions together with a diffusion term.

\section{ACTIVE CONTOURS FOR CT IMAGE TUMOR IDENTIFICATION}

To allow local deformations with a shape constraint that allows local variations around the segmented masks. Its main objective is to produce complete and accurate contour lines of a specific object in the image for a given input image. Let us see for a normal CT image shown in figure 3(a) and tumor CT image in figure $3(\mathrm{~b})$. The encircled dashed lines are where the tumor is located. Figure 3(a) CT image does not have a tumor, i.e. the blood flow is normal and continuous. However, in figure 3(b) the blood flow is abnormal and is discontinues which indicates a presence of tumor.

Here active contour's (snakes) helps to differentiate these two images by detecting the tumor in the image which containing Contour. Then identifying tumor in the image where it is actually present in the image was the concept of this project. Through Shape segmentation is shows defining a mask by a definite shape whether it is a circle, square, rectangle, or else considering a particular regional shape for further contouring. A snake falls into the closest local energy minimum. The local minima of the snake energy comprise the set of alternative solutions. A higher level knowledge is needed to choose the correct one from the solutions of High-level reasoning and User interaction. These high-level methods can interact with the contour model by pushing it toward to an appropriate local minimum.

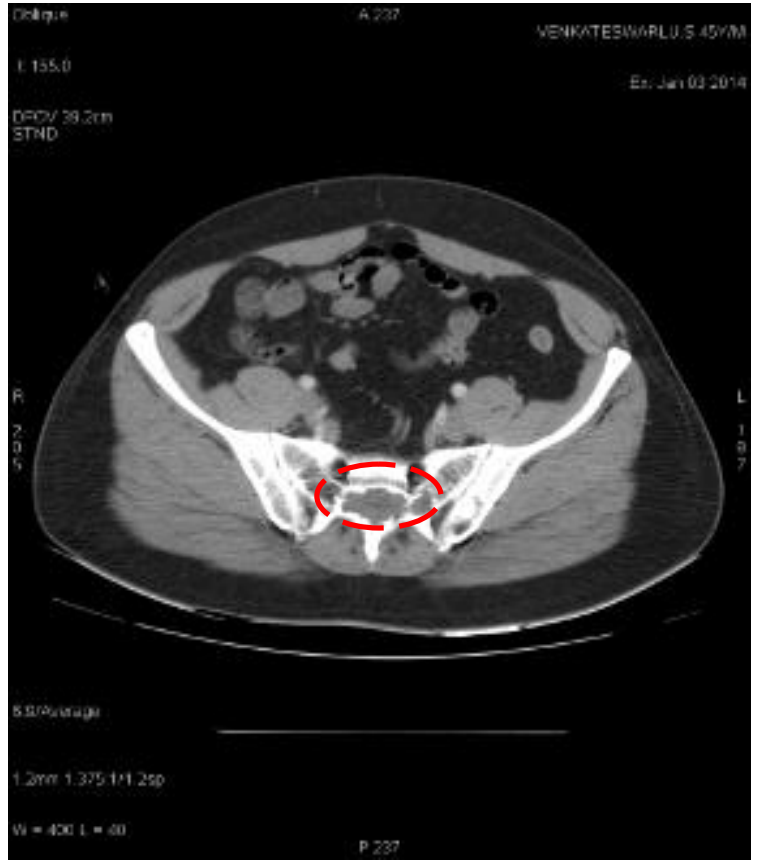

Figure 3(a): CT Image of NormalHealthy patient

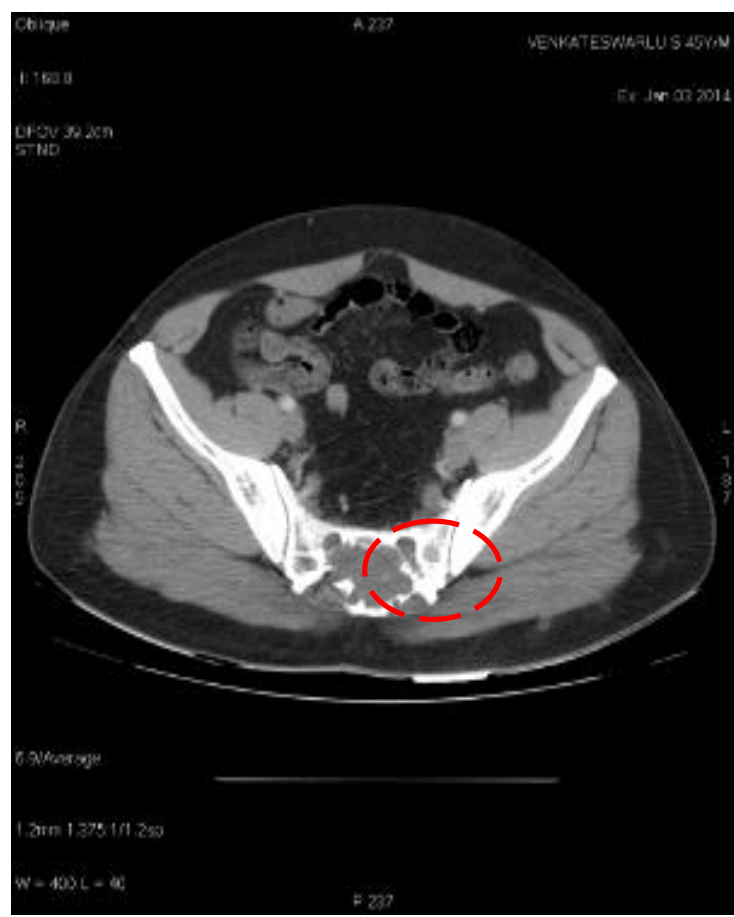

Figure 3(b): CT Image of Diseaced Patient

\section{RESULTS AND DISCUSSION}

Matlab 10 is used for implementing the proposed algorithm. This research used three snake models to accomplish the task. They are active contours, local region based segmentation and level sets model. Figure 4 shows CT medical images used for testing our proposed models. All the models were tested under three noise models such white noise, Gaussian noise and Poisson noise. This method calls for choosing a initial contour close to the area of the tumor and hence a human intervention at the initial stages of detection is mandatory to operate the software. 
The proposed active contour models are applied on the following CT images. The active contours general model outputs are shown in figure 5. The active contours model is applied to normal CT images of a healthy male patient and that of a unhealthy patient having tumor under different situations. The results show visually active contours are not very efficient in identifying the tumor area in the CT scans.

From figure 5 it can be observed clearly that the active contour model fails to identify exact boundaries of the tumor to precision. The comparison with the segmented areas of non tumor CT images reveals that there is very little difference in the segmented areas. The failure of the active contour method is due to the fact that the contour has no prior information regarding the local information near to the tumor. This problem is solved effectively by supplying local information of the $\mathrm{CT}$ image near to the initial contour. Information local to the initial contour propels the contour exactly towards the destination forces in the CT image. Figure 6 shows the responses of experimentation of local region based segmentation.
Figure 6 shows the quality of region based segmentation. All the tumors are detected near perfectly. Comparison with the non tumor images reveals that the CT image segmented areas are entirely different. It can also be observed that the CT image tumor identification is near perfect under various noisy conditions. Finally experiments were conducted on level sets method of segmentation. The results of this technique are shown in figure 7.

Figure 7 show the performance is good but it fails to detect the finer details of the tumor when compared to the previous method of detecting tumors using local regions information. The detection is good but not a perfect detection. This is also same in case of noise added CT images. Level sets are a good prospect if again shape prior information is supplied to the contour.

The observations highlight the best model for detecting tumors in $\mathrm{CT}$ images. From the three models proposed in this research we pin down on Local region based segmentation for CT image tumor detection.

NORMAL IMAGES :

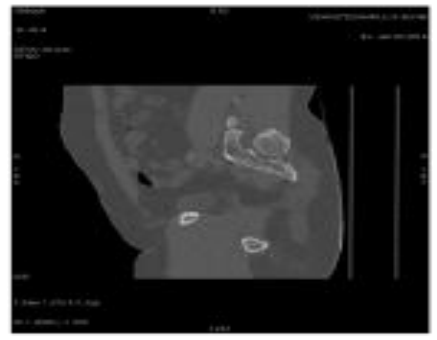

FIG.1A

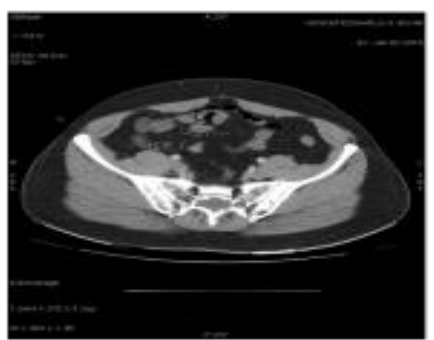

FIG.1B

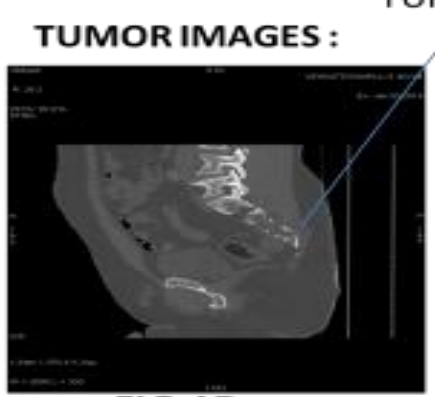

FIG.1D

UMOR AREA

TUMOR AREA

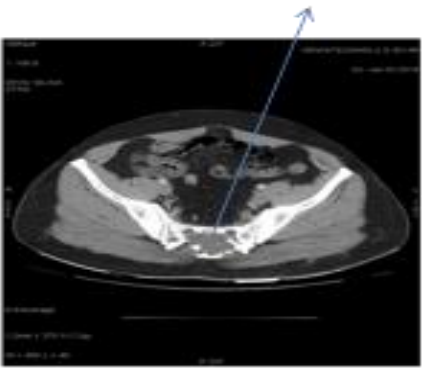

FIG.1E

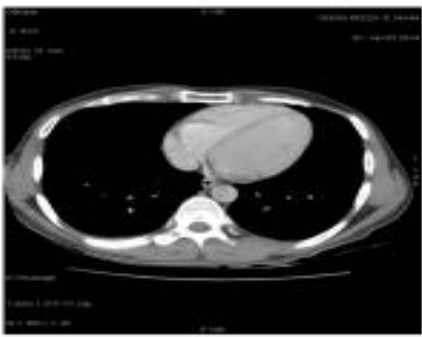

FIG.2C

TUMOR AREA

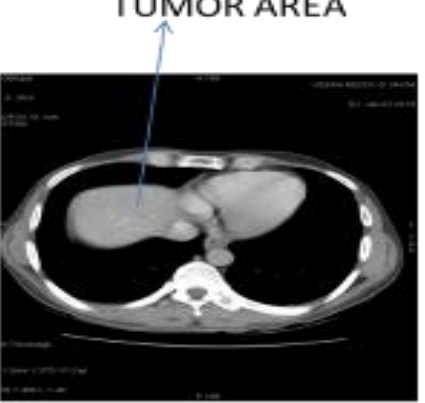

FIG.1F

Figure 4: Test CT Images with and Without Tumor for Experimentation 


\title{
ACTIVE CONTOUR
}

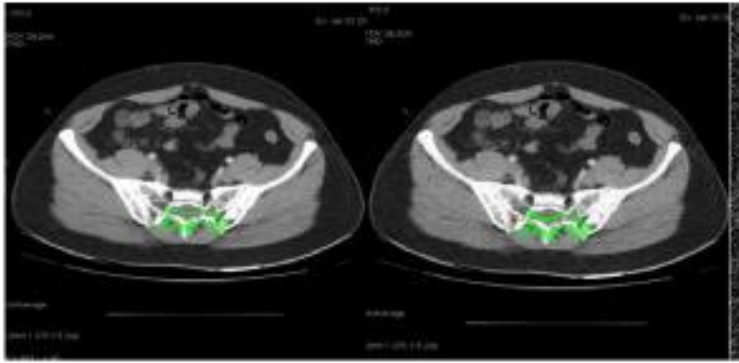
2a. Normal image
2b.normal image with No noise
with white noise

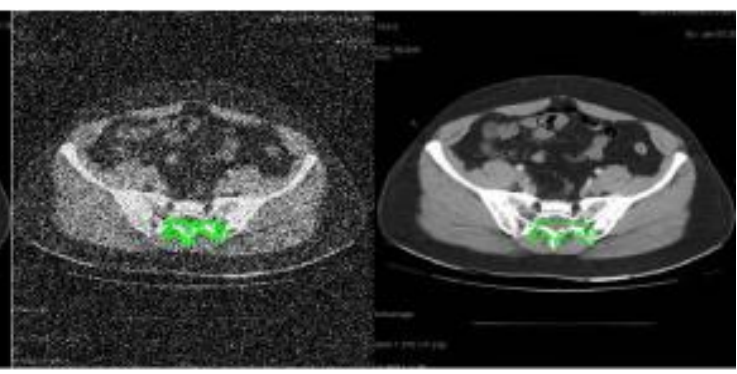

\begin{abstract}
2c.normal image with guassian noise
\end{abstract}

d.normal image with poisson noise

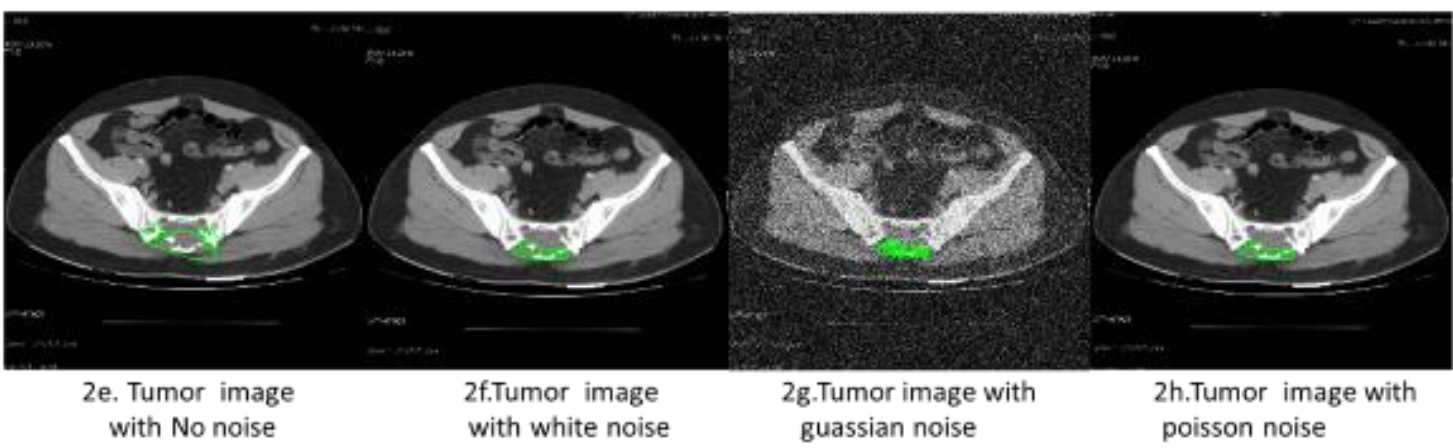

Figure 5: Showing results of proposed active contour model for tumor identification in CT images

\section{LOCAL REGION BASED SEGMENTATION}
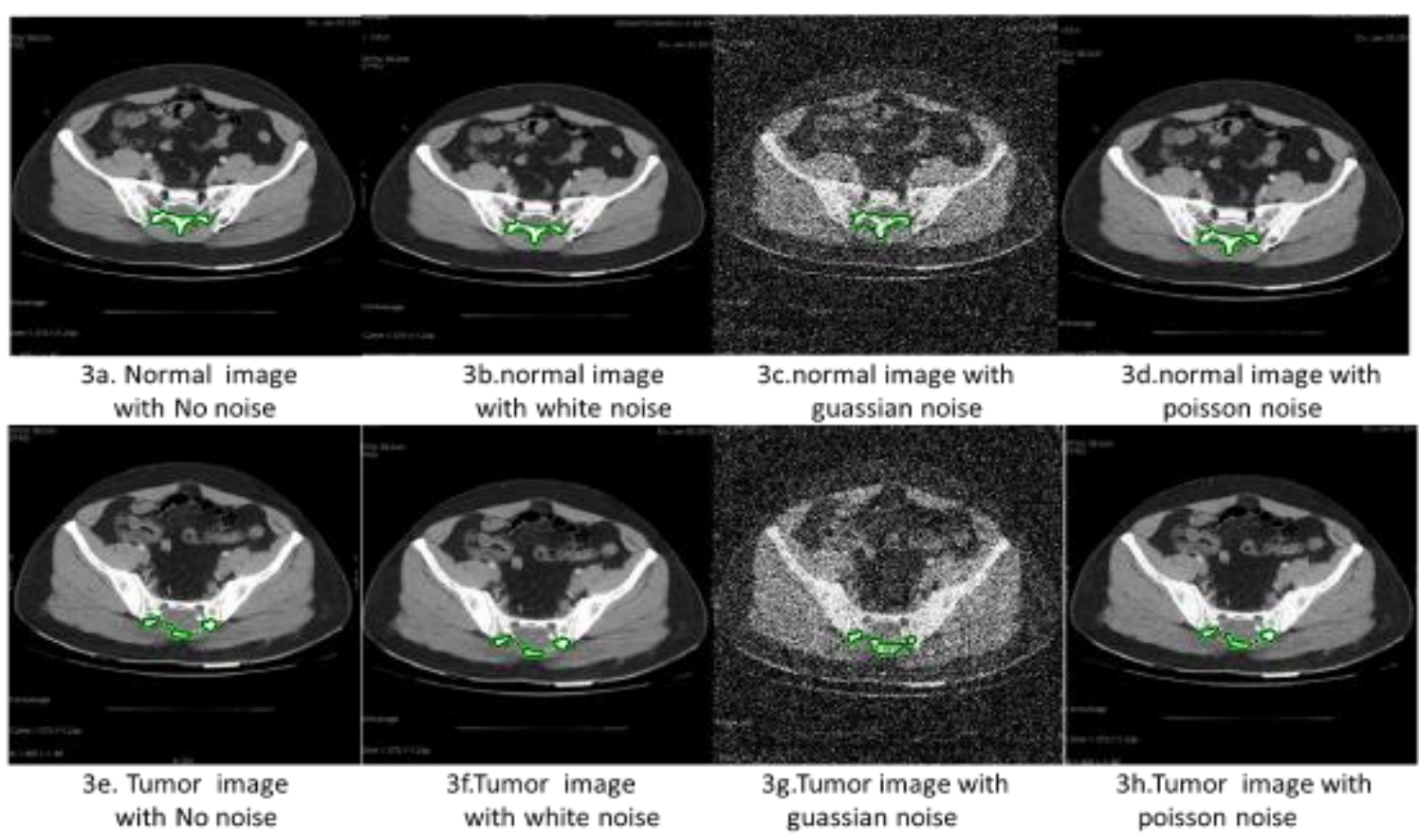

Figure 6: CT Tumor detection using Local Region Based Segmentation 


\section{LOCAL REGION BASED SEGMENTATION}
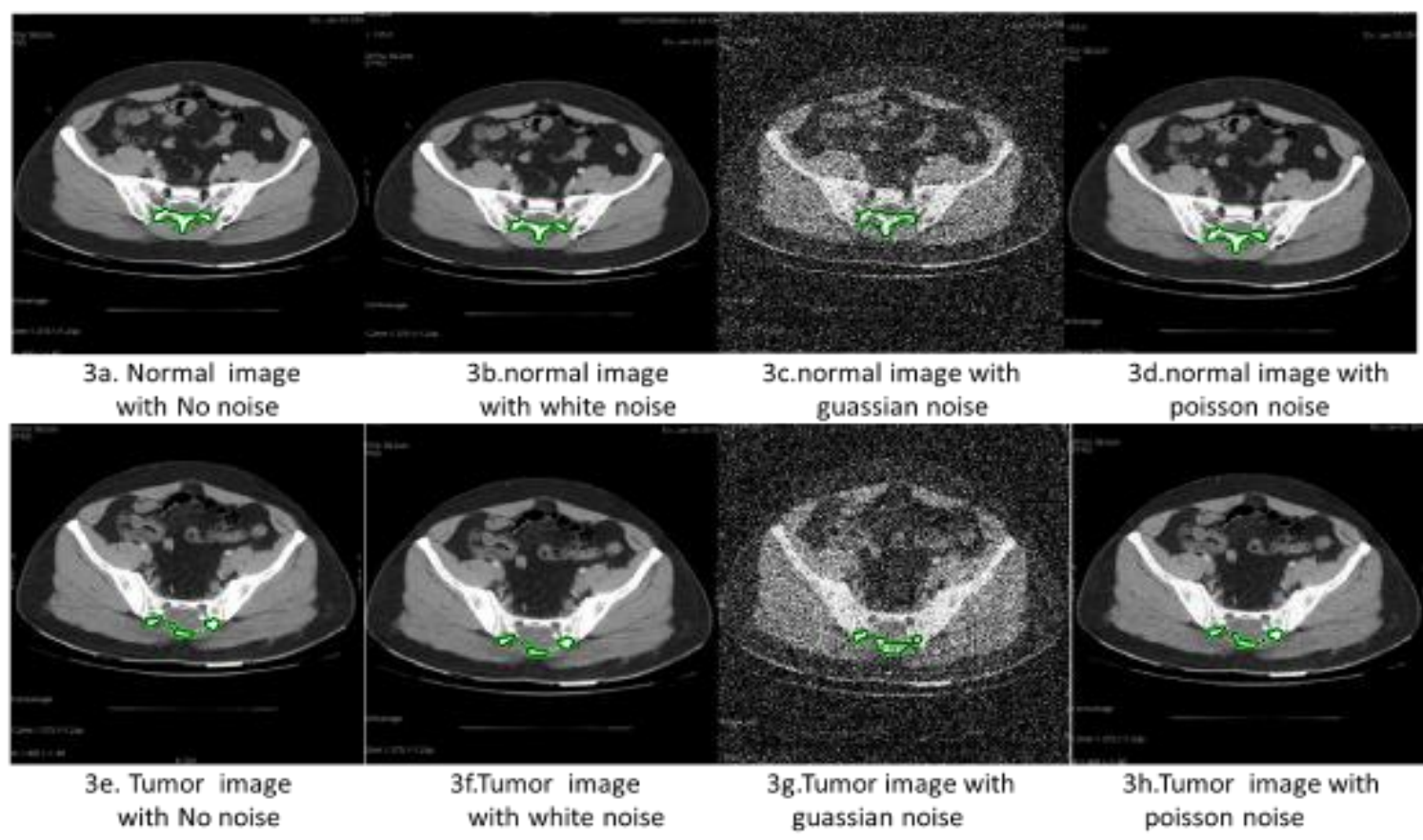

\section{CONCLUSION}

In this paper three methods are proposed to segment a CT medical image tumor. Active contours, Local region based segmentation and level sets methods are used to detect tumor in CT medical images. Active contours are a powerful deformable models used for medical image segmentation. With this research we have demonstrated the use of active contour models can be used to detect tumors in CT medical images. From the three models proposed for CT medical image tumor detection local region based segmentation produces excellent detection followed by level sets to a certain extent. Normal active contours model fails to detect tumors in CT medical images.

\section{REFRENCES}

[1] Maintz, J. B., and Max A. Viergever. "A survey of medical image registration. "Medical image analysis 2.1 (1998): 136.

[2] McAuliffe, Matthew J., et al. "Medical image processing, analysis and visualization in clinical research." ComputerBased Medical Systems, 2001. CBMS 2001. Proceedings. 14th IEEE Symposium on. IEEE, 2001.

[3] Lehmann, Thomas Martin, Claudia Gonner, and Klaus Spitzer. "Survey: Interpolation methods in medical image processing." Medical Imaging, IEEE Transactions on 18.11 (1999): 1049-1075.

[4] Studholme, Colin, Derek LG Hill, and David J. Hawkes. "Automated 3-D registration of MR and CT images of the head." Medical image analysis 1.2 (1996): 163-175.

[5] D. Terzopoulos and K. Fleischer (1988) "Deformable models”. The Visual Computer, Vol.4 No.6, pp306-331.

[6] P.V.V.Kishore, P.Rajesh Kumar, "Sign Language Video Segmentation with Level Sets Fusing Color, Texture, Boundary and Shape Features" Signal \& Image Processing an International Journal, AIRCCSE Publishers, SIPIJ, June 2012, Vol(3), No.(3), ISSN: 2229 - 3922, pp73-92.

[7] P.V.V.Kishore, P.Rajesh Kumar, "Tracking Complex Movements Under Assorted Backgrounds in Sign Language Using Active Contour Models" CiiT International Journal of Digital Image Processing, ISSN 0974 - 9586, Vol(4),Issue(6), DOI: DIP062012009.

[8] P.V.V.Kishore, P.Rajesh Kumar, " Segment, Track, Extract, Recognize and Convert Sign Language Videos to Voice/Text" International Journal of Advanced Computer Science and Applications (IJACSA) ISSN(Print)-2156 5570, ISSN(Online)- 2158-107x, June 2012 Edition Vol(3), No 6, pp35-47.

[9] Luminita Vese and Tony Chan.(2002) "A multiphase level set framework for image segmentation using the mumford and shah model". International Journal of Computer Vision, Vol.50,No. 3 pp271-293.

[10] Pelizzari, Charles A., George TY Chen, Danny R. Spelbring, Ralph R. Weichselbaum, and Chin-Tu Chen. "Accurate three-dimensional registration of CT, PET, and/or MR images of the brain." Journal of computer assisted tomography 13, no. 1 (1989): 20-26.

[11] Qing-song Zhu, Yao-qin Xie, Lei Wang (2010) Video Object Segmentation by Fusion of Spatio-Temporal Information Based on Gaussian Mixture Model, Bulletin of advanced technology research, vol. 5, No. 10, pp38-43.

[12] Wen-kai Tsai, Chung-chi Lin, Shyue-wen Yang, Ming-hwa Sheu, Ching-lung Su (2008), "Adaptive Motion Gesture Segmentation”. The 2008 International Conference on Embedded Software and Systems Symposia (ICESS2008), pp386-391. 
[13] N. Peyrard and P. Bouthemy (2002) "Content-based video segmentation using statistical motion models". In Proc. $B M V C$, Cardiff, pages 527-536.

[14] Ginmo Chung and Luminita Vese. (2003) "Image segmentation using a multilayer level set approach. Technical Report" 03-53, UCLA.

[15] Armato III, Samuel G., and William F. Sensakovic. "Automated lung segmentation for thoracic CT: Impact on computer-aided diagnosis1." Academic Radiology 11, no. 9 (2004): 1011-1021.

[16] Prastawa, Marcel, Elizabeth Bullitt, Sean Ho, and Guido Gerig. "A brain tumor segmentation framework based on outlier detection." Medical Image Analysis 8, no. 3 (2004): 275-283.
[17] Sluimer, Ingrid, Mathias Prokop, and Bram van Ginneken. "Toward automated segmentation of the pathological lung in CT." Medical Imaging, IEEE Transactions on 24, no. 8 (2005): 1025-1038.

[18] P.V.V.Kishore, P.Rajesh Kumar, "Segment, Track, Extract, Recognize and Convert Sign Language Videos to Voice/Text" International Journal of Advanced Computer Science and Applications (IJACSA) ISSN(Print)2156 5570, ISSN(Online)- 2158-107x, June 2012 Edition Vol(3), No 6, pp35-47.

[19] P.V.V.Kishore, S.R.C.Kishore, M.V.D.Prasad, "Conglomeration of Hand Shapes and Texture Information for Recognizing Gestures of Indian Sign Language Using Feed forward Neural Networks", International Journal of engineering and Technology (IJET), ISSN: 0975-4024, Vol 5 No 5 Oct-Nov 2013, pp3742-3756. 